\title{
ESTILOS DE APRENDIZAJE Y RENDIMIENTO ESCOLAR EN UN COLEGIO PARTICULAR DE LIMA NORTE
}

\section{Learning styles and school performance in a particular school of Lima Norte}

\author{
Elsa María Bontempo Lozano*
}

\begin{abstract}
Resumen
Objetivo: Identificar si existe una relación significativa entre estilos de aprendizaje y rendimiento escolar en el área de razonamiento matemático.

Método: El estudio se llevó a cabo con 112 alumnos de primero de secundaria del colegio particular "El Buen Pastor" del distrito de Los Olivos. Elegidos de manera intencional, por ser no probabilística. Se realizó una investigación cuantitativa, de tipo observacional, básica, prospectiva, transversal y correlacional, se utilizó el Test de Estilos de Aprendizaje de Felder y Silverman.

Resultados: Existe una mayor predominancia en los EA Activo, Visual y Secuencial, encontrándose que en la categoría secuencial-global existen diferencias significativas, podría haber una relación directa con $R A$ en razonamiento matemático. Se realizó el análisis estadístico paramétrico donde los datos tenían una distribución normal.

Conclusiones: Los EA son significativos para el proceso de enseñanza-aprendizaje, no hay diferencias altamente significativas ni directas con el RA.
\end{abstract}

Palabras clave: Estilos de Aprendizaje; Rendimiento Académico.

\begin{abstract}
Objective: To identify if there is a significant relationship between learning styles and school performance in the area of mathematical reasoning.

Method: The study was carried out with 112 first year high school students from the non-state school "El Buen Pastor" in the Los Olivos district. Intentionally chosen, as it is non-probabilistic. A quantitative, observational, basic, prospective, cross-sectional and correlational research was carried out; the Felder and Silverman Learning Styles Test was used.

Results: There is a greater predominance in Active, Visual and Sequential AE, finding that the sequentialglobal category exists significant differences, there could be a direct relationship with AR in mathematical reasoning. Parametric statistical analysis was performed where the data had a normal distribution.

Conclusions: AE are significant for the teaching-learning process, there are no highly significant or direct differences with AR.
\end{abstract}

Keywords: Learning Styles; Academic performance.

* Docente de la Universidad César Vallejo. Directora del Centro Psicopedagógico. Ps.bontempos.consultora@gmail.com, Lima - Perú. ORCID: 0000-0003-0661-7952

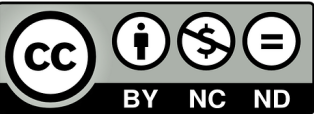

Para utilizar este artículo debe incluir: Autor / Título / ISSN. 


\section{INTRODUCCIÓN}

La UNESCO (2007), menciona que nuestra sociedad presenta serios problemas de rendimiento académico, en los alumnos latinoamericanos el rendimiento es bajo y solo siete países incluido Chile están por debajo de los países europeos y del este asiático. Perú es el país con mayor porcentaje de estudiantes que no superan el promedio establecido por la OCDE tanto en lectura $(60 \%)$ como en ciencia $(68,5 \%)$. Además, es el segundo peor situado en matemáticas $(74,6 \%)$, solo por detrás de Indonesia. (Citado por BBC Mundo, 2016).

Para Armstrong (1999), los maestros tienen un rol de acompañante en descubrir el tipo de estilo de aprendizaje que muestra el alumno; a principios del siglo XXI los pedagogos se muestran sensibilizados, con la idea de considerar al alumno como polo activo del proceso enseñanza- aprendizaje con la intención de lograr mayor efectividad en la misma y de transitar irremediablemente por una mejor enseñanza, más clara y exhaustiva compresión del aprendizaje y de lo que podrá aprender.

Es importante considerar que los Estilos de Aprendizaje juegan un rol principal dentro del proceso enseñanza/aprendizaje durante todo el proceso de su vida académica y profesional, ya que se considera un proceso de cambio relativamente permanente en el comportamiento del ser humano y éste es generado por una experiencia (Feldman, 2005).

Estudios como los de Dunn, R; Dunn, R. y Price, G., (1979, p. 41) han demostrado que el proceso de aprendizaje frente a sus estilos tiene una relación causaefecto entre lo cognitivo y los estilos de aprendizaje y menciona que "la manera en que los estímulos básicos afectan a la habilidad de una persona para absorber y retener la información" mientras que para Hunt (1979, p. 27) estos "describen las condiciones bajo las que un discente está en la mejor situación para aprender, o qué estructura necesita el discente para aprender mejor".

Por otro lado, Schermeck, R. (1992, p. 80) refiriéndose a los estilos de aprendizaje señala: "son simplemente el estilo cognitivo que un individuo manifiesta cuando se enfrenta a una tarea de aprendizaje y refleja las estrategias preferidas, habituales y naturales del estudiante para aprender, para que pueda ser ubicado en algún lugar entre la personalidad y las estrategias de aprendizaje, por no ser tan específicos como estas últimas, ni tan general como la primera”.

Esta investigación toma como referencia que los estilos de aprendizaje son la forma o estilos de cómo el alumno aprende y recepciona los conocimientos; aunque algunos investigadores intentan explicar sí realmente esto influye en el rendimiento académico, para poder explicar si la investigación se usa como instrumento para la medición del estilo de aprendizaje propuesto por Felder y Silverman (Test de estilos de aprendizaje), aplicado a estudiantes de la Universidad Tecnológica de Bolívar, donde se encontró que es estadísticamente significativa la relación entre los estilos de aprendizaje reflexivo, verbal y secuencial y los resultados académicos de los estudiantes, lo que significaría que el papel del maestro no solo está relacionado con el conocimiento de las materias sino también con las estrategias didácticas en la práctica docente, en relación con los estilos de aprendizaje de los estudiantes (Huertas y Garcés, 2003).

Es importante mencionar que el Ministerio de Educación en el año 2018, presenta resultados de la Evaluación Censal (ECE) de estudiantes de $4^{\circ}$ de primaria dónde se identificó que el área de matemática, el $24.2 \%$ se encuentra en inicio del logro esperado, mientras que el grado de $2^{\circ}$ de secundaria el $33.7 \%$ que se ubica en el nivel de logro inicial tanto para las escuelas públicas y privadas a nivel nacional, siendo los resultados de gran preocupación, es importante el análisis y la búsqueda de las causantes ante esta dificultad.

Esta evaluación se viene realizando desde el año 2007, siendo sólo el año 2017 suspendida por el fenómeno del niño costera y la huelga magisterial, que se le aplica a los alumnos de $4^{\circ}$ de primaria y de $2^{\circ}$ de secundaria en los meses de octubre y noviembre a quienes se les evalúa las áreas de matemática, CTA, lectura, historia y geografía y economía, siendo un gran aporte a nuestro país y buscando conocer la realidad en la que nos encontramos.

Por lo tanto, esta investigación servirá para esclarecer la relación significativa que puede existir entre ambas variables; tomando en cuenta que el objetivo general de la presente investigación tiene como finalidad analizar el nivel de asociación entre los estilos de aprendizaje y el rendimiento escolar 
en el área de matemática en alumnos de primero de secundaria de una Institución Educativa no estatal.

\section{MÉTODO}

\section{Diseño y participantes:}

Se utilizó una investigación cuantitativa, de tipo observacional, básica, prospectiva, transversal y descriptivo- comparativo.

La población estuvo constituida por 112 alumnos del primer grado de secundaria, 58 mujeres y 54 varones, que oscilan entre los 11 y 12 años de edad, pertenecientes al Centro Educativo No Estatal "El Buen Pastor" del distrito de Los Olivos.

Se obtuvo el permiso de la institución educativa para la aplicación del instrumento a los alumnos y para la obtención de las notas de fin de año de los alumnos seleccionados para la ejecución del estudio.

Por otro lado, los padres tuvieron conocimiento que sus hijos deberían ser evaluados en sus horas de clases para la obtención de la muestra del estudio.

A todos los participantes se les aplicó el proceso de consentimiento informado que consiste en ser informados de los objetivos del estudio.

\section{Instrumentos:}

Para la presente investigación se utilizaron dos instrumentos, la prueba de Estilos de Aprendizaje de Felder y Silverman y el Registro de notas del promedio final de razonamiento matemático que se describen a continuación:

a) Se utilizó el Inventario de Estilos de Aprendizaje de Felder y Silverman, el cual tiene una aplicación Colectiva, para todo el nivel de secundaria y con la duración de 50 a 60 minutos, cuya finalidad es conocer el tipo de Estilos de Aprendizaje en las dimensiones (Activo - Reflexivo, Sensitivo - intuitivo, visual - verbal, secuencial - Global), tiene un manual y protocolo de respuesta.

La validación de la prueba de Estilos de Aprendizaje de Felder y Silverman fue hecha utilizando el Índice de Lawshe (1975). Por lo cual previamente se solicitó a seis expertos la revisión de la prueba del Inventario de Felder y Silverman referente a los estilos de aprendizaje.

El análisis de ítems se estableció a través de la correlación ítem - test corregido y la confiabilidad se estudió con el método de consistencia interna del Alfa de Cronbach.

La Aplicación y Calificación parte de cuatro escalas bipolares relacionadas con las preferencias en el estilo de aprendizaje. Estas escalas son determinadas por un cuestionario de 44 preguntas, que están relacionadas con situaciones hipotéticas del individuo y su aprendizaje. Sin embargo, si se observa más adelante las categorías que se utilizan en este modelo son un total de 19 categorías, las cuales son el resultado y se obtienen de la siguiente forma:

Cada pregunta tiene solo dos opciones de respuesta "a" o "b". El encuestado selecciona solamente una respuesta para cada pregunta, la cual aporta un puntaje en la categoría correspondiente, así por ejemplo las preguntas 1, 5, 9, 13, 17, 21, 25, $29,33,37$ y 41 corresponden a la categoría activo o reflexivo, si la respuesta en la pregunta 9 fue $\mathrm{A}$, se coloca 1 en la casilla debajo de la letra A y al lado derecho de la pregunta 9 .

Posteriormente se suma cada columna, obteniendo un total. Por ejemplo, en el caso de la categoría bipolar Activo - Reflexivo, se obtiene 7 para A y 4 para B, luego se resta BA (4-7), es decir obteniendo un resultado de -3 , que determinará el lugar del individuo en la siguiente escala (Tabla 3) para cada una de las categorías.

De tal manera que, un individuo que en las cuatro escalas bipolares de Felder se encuentre ubicado entre -3 y 3 , se clasifica como bien Balanceado o Equilibrado; mientras que otro que, verbigracia, en una de las categorías tenga una fuerte preferencia se clasifica como se denomine dicha categoría; por ejemplo, en el caso que se presenta, sería un individuo Secuencial. Aquellos que se encuentren dentro de los puntajes -5 y -7 se clasificarán como Moderados A mientras los que estén entre 5 y 7 serán Moderados B. 
b) Para realizar la correlación de las notas se utilizó el registro de notas del grado, se tomó las notas del promedio final del curso de matemática que otorgó la dirección del Centro Educativo.

\section{Análisis de datos:}

Estos participantes fueron elegidos de manera intencional, por lo que la muestra es de carácter no probabilística.

Se realizó el análisis estadístico paramétrico donde se evidenció que los datos tenían una distribución normal, por ello se usó la T de Student para comparar la media de los problemas.

\section{RESULTADOS}

Se evidenció que existe un mayor porcentaje y predominancia en los estilos de aprendizaje Activo, Visual y Secuencial como se puede evidenciar en la Tabla 1.

\section{Tabla 1}

Frecuencia de Estilos de Aprendizaje

\begin{tabular}{lcc}
\hline Categoría & Frecuencia & Porcentaje \\
\hline Activo & 90 & 80,4 \\
Reflexivo & 22 & 19,6 \\
Sensitivo & 59 & 52,7 \\
Intuitivo & 53 & 47,3 \\
Visual & 90 & 80,4 \\
Verbal & 22 & 19,6 \\
Secuencial & 74 & 66,1 \\
Global & 38 & 33,9 \\
\hline
\end{tabular}

Al realizar el análisis del promedio de notas obtenidas al final del año escolar en el curso de razonamiento matemático (Tabla 2), se evidencia una mayor predominancia entre las notas de 13 y 14 , según el DCN (2016) lo ubica en la categoría de B (en proceso), que hace relación que el estudiante está en camino de lograr los aprendizajes previstos, para lo cual requiere acompañamiento durante un tiempo razonable para lograrlo y cumplir con los lineamientos del Diseño Curricular Nacional.
Tabla 2

Frecuencia de notas en Raz. Matemático

\begin{tabular}{ccc}
\hline Notas & Frecuencia & Porcentaje \\
\hline 9 & 1 &, 9 \\
10 & 4 & 3,6 \\
11 & 5 & 4,5 \\
12 & 19 & 17 \\
13 & 24 & 21,4 \\
14 & 20 & 17,9 \\
15 & 13 & 11,6 \\
16 & 13 & 11,6 \\
17 & 9 & 8 \\
18 & 3 & 2,7 \\
19 & 1 &, 9 \\
\hline
\end{tabular}

Por otro lado, en la categoría Activo - Reflexivo, el puntaje obtenido $\mathrm{p}=, 644$ que es mayor al 0.05 por lo que se puede indicar que no existen diferencias estadísticamente significativas con el rendimiento académico en razonamiento matemático. (Tabla 3).

Tabla 3

Diferencias en el Rendimiento Académico de Raz. Matemático según el estilo Activo- Reflexivo

\begin{tabular}{llllllll}
\hline Estilo 1 & $\mathrm{N}$ & Media & $\begin{array}{c}\text { Desviación } \\
\text { estándar }\end{array}$ & $\begin{array}{c}\text { Media } \\
\text { de error } \\
\text { estándar }\end{array}$ & $\mathrm{t}$ & $\mathrm{gl}$ & $\begin{array}{c}\text { Sig. } \\
\text { (bilateral) }\end{array}$ \\
\hline Activo & 90 & 13,82 & 1,964 &, 207 &,- 464 & 110 &, 644 \\
Reflexivo & 22 & 14,05 & 2,257 &, 481 & & & \\
\hline
\end{tabular}

En la categoría Sensitivo - Intuitivo, el puntaje obtenido $\mathrm{p}=, 136$ es mayor al 0.05 por lo que se puede indicar que no existen diferencias estadísticamente significativas con el rendimiento académico en razonamiento matemático. (Tabla 4) 
Tabla 4

Diferencias en el Rendimiento Académico de Raz. Matemático según el estilo Activo- Reflexivo

\begin{tabular}{lccccccc}
\hline Estilo 2 & $\mathrm{N}$ & Media & $\begin{array}{c}\text { Desviación } \\
\text { estándar }\end{array}$ & $\begin{array}{l}\text { Media } \\
\text { de error } \\
\text { estándar }\end{array}$ & $\mathrm{t}$ & $\mathrm{gl}$ & $\begin{array}{c}\text { Sig. } \\
\text { (bilateral) }\end{array}$ \\
\hline Sensorial & 59 & 14,14 & 1,934 &, 252 & 1,501 & 110 &, 136 \\
Intuitivo & 53 & 13,57 & 2,080 &, 286 & & & \\
\hline
\end{tabular}

En la categoría Visual - Verbal, el puntaje obtenido $\mathrm{p}=, 485$ es mayor al 0.05 por lo que se puede indicar que no existen diferencias estadísticamente significativas con el rendimiento académico en razonamiento matemático. (Tabla 5)

\section{Tabla 5}

Diferencias en el Rendimiento Académico de Raz. Matemático según el estilo Visual - Verbal

\begin{tabular}{lccccccc}
\hline Estilo 3 & $\mathrm{N}$ & Media & $\begin{array}{c}\text { Desviación } \\
\text { estándar }\end{array}$ & $\begin{array}{c}\text { Media } \\
\text { de error } \\
\text { estándar }\end{array}$ & $\mathrm{t}$ & $\mathrm{gl}$ & $\begin{array}{c}\text { Sig. } \\
\text { (bilateral) }\end{array}$ \\
\hline Activo & 90 & 13,80 & 2,018 &, 213 &,- 700 & 110 &, 485 \\
Reflexivo & 22 & 14,14 & 2,031 &, 433 & & & \\
\hline
\end{tabular}

Finalmente, en la categoría Secuencial-Global se encontró que existen diferencias estadísticamente significativas, ya que el puntaje $\mathrm{p}=, 029$ es menor al 0.05 por lo que podría existir una significancia directa con el rendimiento académico en razonamiento matemático. (Tabla 6)

\section{Tabla 6}

Diferencias en el Rendimiento Académico de Raz. Matemático según el estilo Secuencial - Global

\begin{tabular}{lccccccc}
\hline Estilo 4 & $\mathrm{N}$ & Media & $\begin{array}{c}\text { Desviación } \\
\text { estándar }\end{array}$ & $\begin{array}{c}\text { Media } \\
\text { de error } \\
\text { estándar }\end{array}$ & $\mathrm{t}$ & $\mathrm{gl}$ & $\begin{array}{c}\text { Sig. } \\
\text { (bilateral) }\end{array}$ \\
\hline Activo & 74 & 14,16 & 1,916 &, 223 & 2207 & 110 &, 029 \\
Reflexivo & 38 & 13,29 & 2,104 &, 341 & & & \\
\hline
\end{tabular}

\section{DISCUSIÓN}

En la presente investigación los hallazgos encontrados dieron como resultado que los estilos de aprendizaje no tienen una relación directa frente a los resultados obtenidos en el rendimiento académico en razonamiento matemático, pero si se encontró que en el estilo Secuencial- Global existe una diferenciación y un grado de significancia directa, lo cual podría indicar que si un docente utiliza un método secuencial, hace relación a explicar bajo pasos y líneas, podrán aprender con mayor predominancia; por otro lado, al tener una enseñanza global, se aprende en grandes bloques absorbiendo material casi aleatoriamente sin ver conexiones; para repentinamente unir estos conocimientos, es importante resaltar que el grado evaluado tiene una mayor predominancia al estilo secuencial, los cuales se sienten cómodos con el material presentado en una progresión lógica ordenada dominar, el aprendizaje como el educador presenta. Siguen los procesos de razonamiento lineal en la resolución de problemas, y pueden trabajar con el material incluso cuando sólo tienen una comprensión parcial o superficial de la misma. Pueden ser fuertes en el pensamiento convergente y el análisis, y aprender mejor cuando los educadores presentan materiales en una progresión constante de la complejidad y dificultad (Felder y Silverman, 1988, p. 679).

Se pudo identificar que existen diferencias estadísticamente significativas entre los estilos de aprendizaje y el rendimiento académico en el área de razonamiento matemático, no es altamente significativo estadísticamente, ya que el estilo no garantiza un buen desempeño o un alto rendimiento académico, pero si se pudo encontrar que el estilo de aprendizaje Secuencial es el que podría tener una influencia directa frente a la enseñanza o estilo docente para un mayor conocimiento o aprendizaje significativo en el área de razonamiento matemático; por lo tanto, el estilo del docente también será y tendrá un papel indispensable para generar un mejor rendimiento académico.

Felder y Silverman (1988) mencionan que los estudiantes aprenden de diferentes maneras: por el oír y ver; reflexionando y actuando; razonamiento sea lógico o intuitivo; memorizando, visualizando y estableciendo analogías; y, ya sea de manera constante o en pequeños trozos y piezas de gran tamaño (p.674). También defienden que los estilos de enseñanza 
varían, como la preferencia de un educador para dar conferencias o demostrar, o para centrarse en principios o aplicaciones.

Felder (1996) indica que el modelo de FelderSilverman clasifica a los estudiantes como accesorio en una de las siguientes cuatro dimensiones del estilo de aprendizaje:

- Alumnos sensoriales (concreto, práctico, orientado hacia hechos y procedimientos) o aprendices intuitivos (conceptual, innovador, orientado hacia las teorías y significados);

- Los alumnos visuales (prefieren representaciones visuales del material presentado - imágenes, diagramas, diagramas de flujo) o aprendices verbales (preferir explicaciones escritas y orales);

- Estudiantes activos (aprenden trabajando con otros) o estudiantes reflexivos (aprender a pensar las cosas, trabajando solo);

- Aprendices secuenciales (lineal, ordenado, aprender en pequeños pasos incrementales) o aprendices globales (holísticos, pensadores sistémicos, aprenden en grandes saltos) (Felder, 1996, p. 19). Para Alonso, Gallego y Horney (1994) las personas con estilo de aprendizaje activo suelen ser improvisadores, descubridores, arriesgados y espontáneos, además, suelen presentar conductas de creatividad, son novedosos, aventureros y renovadores, características de liderazgo, tienden a retener y entender la información, haciendo algo activo con ellos, discutiéndolo, aplicándolo o explicándolo a otros y gustan más de trabajos grupales, mientras que los visuales recuerdan más por lo que ven, por medio de fotos, diagramas de flujo, líneas de tiempo, películas y demostraciones, finamente, los secuenciales tienden a entender en pasos lineales, con cada paso seguido lógicamente por otro, encuentran soluciones paso a paso.

Esta investigación presenta una limitación de carácter metodológico, en el sentido de la generalización de los resultados, ya que lo hallado solo se podrá aplicar a una muestra con características similares a los participantes en el presente estudio.
Puede decirse, que existen otras limitaciones, tal como por ejemplo que no existe suficiente información acerca de la variable rendimiento escolar y su relación con los estilos de aprendizaje, especialmente cuando se considera el criterio de actualidad de la información en Psicología, la cual es de cuatro años o menos.

Así mismo, puede señalarse como una limitación, el enfoque de las variables en estudio, pues una, el rendimiento académico está planteado de manera cuantitativa, mientras que los estilos de aprendizaje tienen una óptica de carácter cualitativo.

Finalmente, la relación que existe entre los estilos de aprendizaje y el rendimiento escolar encontrados en la tesis es un hallazgo que guarda afinidad con otras investigaciones como las de Kolb (1984), Grau, Marabotto y Muelas (2004), Huertas y Garcés (2003) quienes afirman que estas variables guardan una relación significativa.

\section{CONCLUSIONES}

Los estilos de aprendizaje son importantes para el proceso de enseñanza-aprendizaje, se pudo encontrar que no existe una diferencia altamente significativa ni directa con el rendimiento académico; sin embargo, se encontró que determinados estilos podrían generar un mejor resultado en el procesamiento de la información, tal como se evidencia con el estilo secuencial en relación con el área de razonamiento matemático. El estilo secuencial tiene una relación significativa en el puntaje obtenido en los resultados del promedio final del área de razonamiento matemático.

Se recomienda hacer una investigación adicional para identificar si el estilo de enseñanza también tiene relación con los estilos de aprendizaje, para corroborar si hay una relación directa con el rendimiento académico en el área de razonamiento matemático.

\section{REFERENCIAS}

Armstrong, T. (1999). Las inteligencias Múltiples en el aula. Buenos Aires: Ed. Manantial. 
Alonso, C., Gallego, D. y Honey, P. (1994). Los Estilos de Aprendizaje. Procedimientos de diagnóstico $y$ mejora. Bilbao: Ediciones Mensajero. Universidad de Deusto.

BBC MUNDO (2016) Los países de América Latina "Con peor rendimiento académico" Recuperado de: https://www.bbc.com/mundo/ noticias/2016/02/160210_paises_bajo_ rendimiento_educacion_informe_ocde_bm

Dolores, B. y Espinoza, R. (2014). Artículo Original: Evaluación de la fiabilidad del cuestionario sobre los estilos de aprendizaje de Felder y Soloman estudiantes de medicina. Inv Ed Med 2015, 4(13), 28-35. Recuperado de: http://www.scielo.org.mx/ pdf/iem/v4n13/v4n13a6.pdf

Dunn, R. ; Dunn, K. y Price, G.(1979). Learning Style Inventory (LSI) for Students in Grade 3-12. Price System. Kansas, Estados Unidos.

Felder, RM, y Silverman, LK (1988). Estilos de aprendizaje y de enseñanza en la educación de ingeniería [Versión electrónica]. Ing. Educación, 78 (7), 674-681 Consultado el 24 de julio de 2009. Recuperado de: http: //www4.ncsu.edu./ unity/lockers/users/f/felder/public/Papers/LS1988.pdf

Felder (2004). Manual de estilos de aprendizaje. Recuperado de: http://biblioteca.ucv.cl/site/ colecciones/manuales_u/Manual_Estilos_de_ Aprendizaje_2004.pdf
Feldman, R. (2005). Psicología: Con aplicaciones en países de habla hispana. México: McGraw-Hill.

Grau, Marabotto y Muelas (2004) Posibles aplicaciones de la informatización del CHAEA. Primer Congreso de Estilos de Aprendizaje. Madrid.

Huertas, N. y Garcés, L. (2003). Estilos de aprendizaje y rendimiento académico ¿Importa el estilo de aprendizaje en el rendimiento educativo? Recuperado: http://www. rediberoamericanadepedagogia.com/index.php/ articulos/boletin-803/estilos-de-aprendizaje-yrendimiento-academicopdf/download.

Hunt, D. (1984). Learning Styles and Student's needs: An introduction to conceptual level in "Students Learning: Diagnosing and prescribing Programs". Reston, Virginia.

Ministerio de Educación. (2016). Currículo Nacional de la educación básica regular. Lima: Primera edición.

Schunk, D. (1991). Autoeficacia y Motivación Académica. Educacional Psycocholigist. Estados Unidos. Recuperado de: https://eprints.ucm. es/16670/1/T34002.pdf

UNESCO.(2014). Enseñanza y Aprendizaje: Lograr la calidadpara todos. Recuperadode:http://unesdoc. unesco.org/images/0022/002261/226159s.pdf

Fecha de recepción: 25 de julio 2019

Fecha de aceptación: 27 de agosto 2019 